\title{
Prevalence of low body mass in rheumatoid arthritis: association with the acute phase response
}

\author{
R Munro, H Capell
}

\begin{abstract}
Objective-To ascertain the prevalence of low body mass in a rheumatoid arthritis (RA) population and to explore a possible relation with the acute phase response. Methods-97 patients who fulfilled the American College of Rheumatology (ACR) criteria for RA were recruited. Change in weight from initial presentation was noted. Body mass index (BMI), upper arm fat and muscle areas were recorded together with fat free mass calculated from the waist measurement. Blood samples were taken for erythrocyte sedimentation rate (ESR), C reactive protein (CRP), and serum albumin.

Results-13\% of the RA group fell into the lowest 5th centile for BMI for the general population. The loss of body mass was greater for lean tissue than fat, with over $50 \%$ of the RA group falling into the lowest 10 th centile of a reference population for the upper arm muscle area. Female patients who lost greater than $15 \%$ of their initial weight had higher health assessment questionnaire (HAQ) results than the rest of the group $(p=0.020)$. In female patients there was a significant correlation between reduced fat free mass and the acute phase response $(E S R \mathrm{p}=\mathbf{0 . 0 1 6}$ and CRP p =0.003)

Conclusions-There is an increased prevalence of low body mass, greatest for lean tissue, in the RA population. In the female group there was an inverse relation between the acute phase response and fat free mass. Female patients with RA who lose a significant amount of weight are more disabled as assessed by HAQ.
\end{abstract}

(Ann Rheum Dis 1997;56:326-329)

Rheumatoid arthritis (RA) results in profound weight loss in some people. The association between weight loss and inflammatory joint disease has been recognised since the mid 19th century. ${ }^{1}$ The proportion of RA patients, however, who have significant but subclinical reduction in body mass is less clear.

Weight loss and loss of lean body mass in particular are powerful predictors of health both in disease states and the general population. ${ }^{23}$ Studies in patients with AIDS and chronic obstructive airways disease have shown a strong relation between weight loss and increased morbidity and mortality. ${ }^{45}$ The reasons for weight loss in RA are probably multi-factorial and include mechanical problems leading to muscle wasting, poor appetite because of drug therapy and disease, and the metabolic burden of the inflammatory response. This study was designed to investigate the prevalence of low body mass in an RA population attending a rheumatology clinic and explore the relation, if any, with disease activity.

\section{Methods}

PATIENTS

Ninety six patients who fulfilled the ACR criteria for RA were recruited serially from a return rheumatology outpatient clinic. Patients who were pregnant, not ambulant or taking oral corticosteroids were excluded. Patients with bilateral shoulder surgery or severe shoulder disease were excluded as this may have interfered with the upper arm anthropometry results.

None of the patients had co-morbid medical conditions that are known to affect body mass. Table 1 shows the demographic details of the study group. Functional status was assessed using the Health Assessment Questionnaire (HAQ) score. Social deprivation was assessed from the Carstairs Score. ${ }^{6}$

ANTHROPOMETRY

The subjects were weighed on balance beam scales to the nearest $0.1 \mathrm{~kg}$. Initial weight at time of presentation to a specialist clinic was noted if this was available. Standing height was measured on a wall mounted stadiometer to the nearest centimetre. Body mass index was calculated from: weight $/$ height $^{2}\left(\mathrm{~kg} / \mathrm{m}^{2}\right)$. Knee height was also recorded with the patient seated and both the ankles and knees flexed to ninety degrees. The measurement was taken from the base of the heel pad to the superior pole of the patella. Knee height varies very little with age unlike standing height and is therefore useful in comparing body composition results across age groups.

Skin fold thickness using standard skin fold callipers was measured over the triceps at the midpoint between the acromion and olecranon. Upper arm circumference was taken at the same site. Measurements were made on the right arm unless patients had shoulder disease in which case the least affected arm was used. Upper arm muscle and fat areas were calculated from standard equations using triceps skin fold thickness and upper arm circumference. ${ }^{8}$

Waist circumference to the nearest $0.1 \mathrm{~cm}$ was recorded at the midpoint between the superior iliac crest and lower costal margin. Fat mass was calculated from waist circumference, weight, and age. This method correlates well
Accepted for publication 20 February 1997 
Table 1 Demographic details

\begin{tabular}{lllll}
\hline & \multicolumn{2}{c}{ Men $(n=36)$} & \multicolumn{2}{c}{ Women $(n=61)$} \\
\hline Age (y) & 56 & $(46-60)$ & 52 & $(43-60)$ \\
Disease duration $(\mathrm{y})$ & 10 & $(7-17)$ & 9 & $(5-17)$ \\
HAQ & 1.750 & $(1.250-2.375)$ & \multicolumn{2}{l}{$1.875(1.500-2.125)$} \\
Body mass index & 26.0 & $(21.3-28.4)$ & 23.5 & $(21.5-26.9)$ \\
Waist $(\mathrm{cm})$ & 94.0 & $(81.6-100.2)$ & 77 & $(71.0-86.3)$ \\
CRP $(\mathrm{mg} / \mathrm{l})$ & 15 & $(7-49)$ & 15 & $(0-30)$ \\
ESR $(\mathrm{mm} / \mathrm{lst} \mathrm{h})$ & 28 & $(16-40)$ & 33 & $(11-46)$ \\
Albumin $(\mathrm{g} / \mathrm{l})$ & 44 & $(43-46)$ & 44 & $(42-46)$ \\
\hline
\end{tabular}

Medians and interquartile ranges are shown.

Table 2 Characteristics of women divided by weight change since initial referral

\begin{tabular}{lllll}
\hline & \multicolumn{2}{l}{ Weight change since referral } & \multicolumn{2}{l}{$\begin{array}{l}\text { Mann-Whitney } \\
p \text { Value }\end{array}$} \\
\cline { 2 - 5 } & $>15 \%$ loss $(n=7)$ & $<$ or $=15 \%$ loss $(n=53)$ & NS \\
\hline Age (y) & $52(42-61)$ & 51 & $(43-60)$ & NS \\
Initial BMI & $26.4(19.3-27.4)$ & 24.2 & $(20.9-27.3)$ & 0.009 \\
Actual BMI & $21.5(16.3-21.8)$ & 24.3 & $(22.0-27.2)$ & NS \\
Disease duration (y) & $12(6-17)$ & 10 & $(5-17)$ & 0.020 \\
HAQ & $2.625(1.876-2.875)$ & $1.875(1.500-2.125)$ & 0.011 \\
ESR (mm/1st h) & $53(36-61)$ & 24 & $(9-41)$ & 0.120 \\
CRP (mg/l) & $31(6-63)$ & 14 & $(0-27)$ & 0.003 \\
Albumin (g/l) & $41(41-42)$ & 45 & $(44-46)$ & \\
\hline
\end{tabular}

Medians and interquartile ranges are shown.

with results achieved using underwater weighing ( $r=0.839$ to 0.868 dependent on sex) and is suitable for population studies. ${ }^{9}$

All anthropometry measurements were made by one observer using the same equipment for each patient.

LABORATORY INVESTIGATIONS

$\mathrm{C}$ reactive protein (CRP), erythrocyte sedimentation rate (ESR), and serum albumin were recorded. Seropositivity for rheumatoid factor was ascertained.

\section{Results}

Table 1 shows the demographic details of the patients. Ninety eight per cent of the study group were white. The patients generally had moderately severe disease as can be seen from

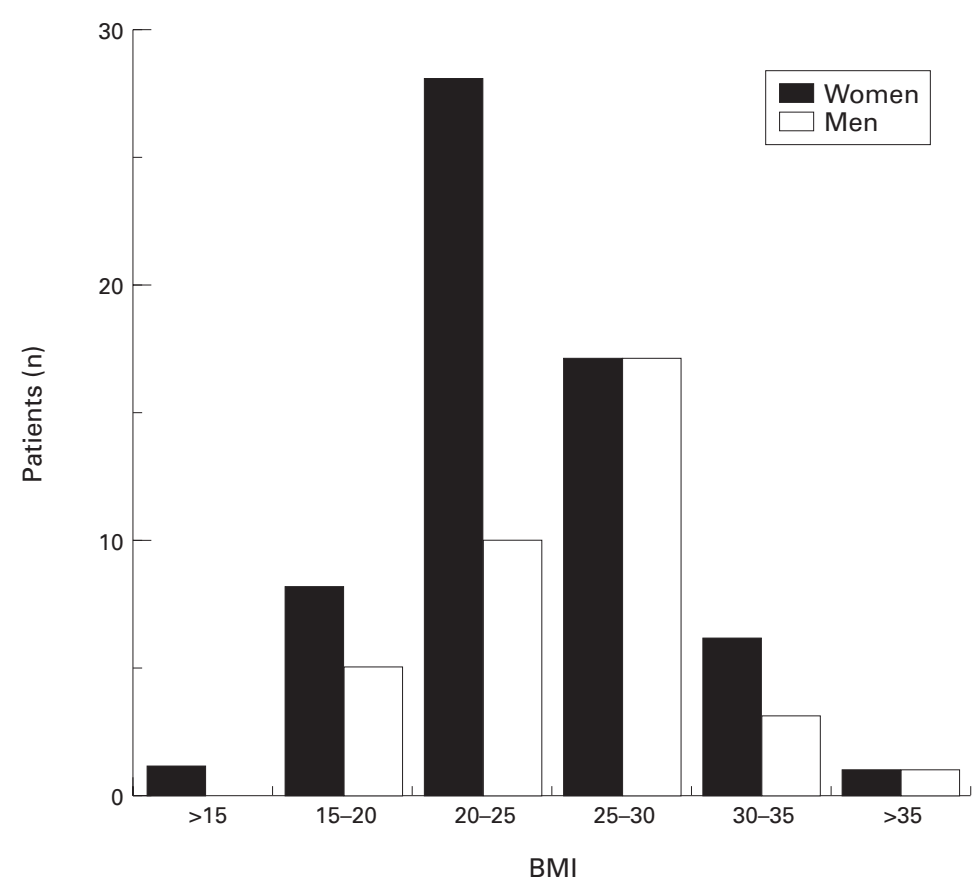

Figure 1 Body mass index distribution. the median HAQ scores. There was a high proportion of seropositive erosive disease in our study group $(87 \%)$, which is representative of the patients attending our review clinics.

BODY MASS INDEX

Figure 1 shows the distribution of BMI in the male and female populations.

Age and sex matched median values of BMI are available for the British population. ${ }^{10}$ In the RA group $13 \%$ of both men and women fell into the lowest 5 th centile for the general population. There was no significant difference in BMI between smokers, ex-smokers, and non-smokers. Patients with low BMI were no more socially deprived than the rest of the patients.

WEIGHT LOSS

Initial weight was available in 60 of 61 women and 34 of 36 men. Thirteen per cent of the women had lost more than $15 \%$ of their initial weight compared with only $3 \%$ of the men. The women who had lost $>15 \%$ of their initial weight were significantly different from the rest of the female group in a number of ways (table 2). Despite being of a similar age and having a similar duration of disease they were significantly more disabled judging from their HAQ score. They also had significantly higher ESRs and lower serum albumin. No comment could be made regarding the male group as only one patient had lost $>15 \%$ of their initial weight.

Weight gain of $>15 \%$ was seen in six women and four men. Their acute phase response and HAQ score was no different from the rest of the cohort.

\section{FAT AND MUSCLE MASS}

Age and sex reference ranges for upper arm muscle and fat areas are available from a large study of 19097 white subjects. ${ }^{11}$

Figure 2 shows the percentage distribution of the RA patients compared with the expected percentile distribution of the control population. The distribution of upper arm fat area is very similar to the normal population. The distribution of muscle mass however is skewed to the left with $50 \%$ of the RA group falling into the lowest 10 th centile for muscle mass of the reference population.

ACUTE PHASE RESPONSE AND LEAN BODY MASS Fat free mass was calculated as outlined in the methods section. To allow for differences in stature a fat free mass index (FFMI) was calculated from fat free mass/knee height $(\mathrm{kg} / \mathrm{cm})$. In women reduced FFMI correlated moderately well with raised acute phase response (ESR $r=-0.323, \mathrm{p}=0.016$ and CRP $r=-0.413, \mathrm{p}=0.003)$ and serum albumin $(r=0.377, \mathrm{p}=0.008)$ when the results were adjusted for age. There was no significant correlation with HAQ $(r=-0.222, \mathrm{p}=0.094)$ or disease duration $(r=-0.150, \mathrm{p}=0.261)$. There was no relation between any of the above variables and low fat free mass index in the male group. 


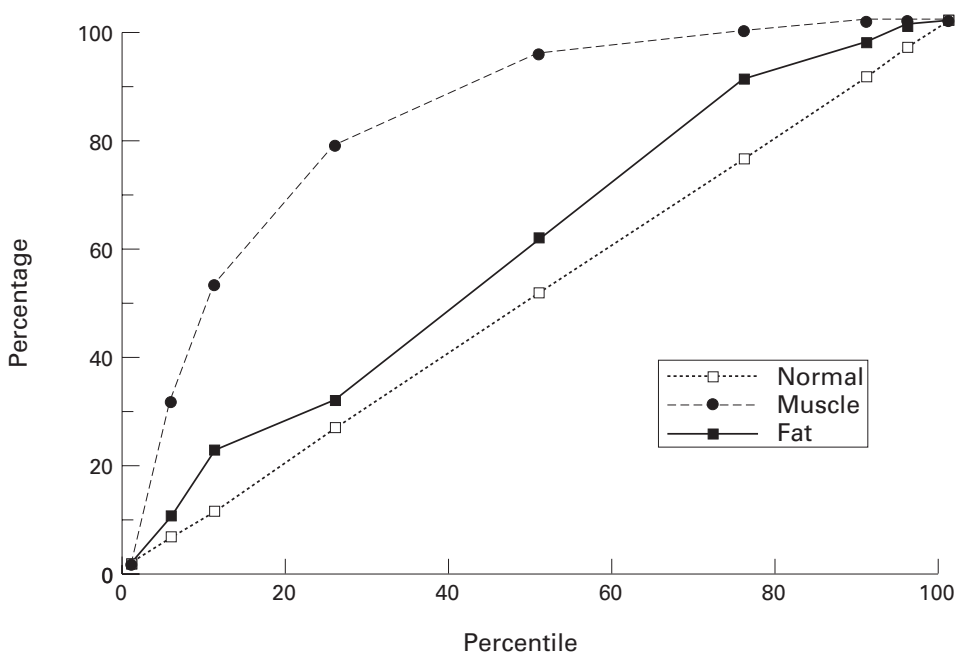

Figure 2 Arm muscle and fat area percentiles versus normal controls.

\section{Discussion}

The results of this study suggest that the prevalence of low body mass in patients with RA may be under recognised. If one uses a definition of being underweight as the lowest 5 th centile of a reference population (possibly an overgenerous cut off point) one in eight RA patients would be considered underweight compared with one in 20 of the general population.

Our results from upper arm anthropometry suggest that the reduction in body mass is greatest for lean tissue and that fat mass is comparatively well maintained. It is striking that over $50 \%$ of the RA group had arm muscle mass areas in the lowest 10th centile of the reference population. Muscle wasting resulting from local joint disease may be partly responsible. These results are very similar, however, to those in a previously published smaller study of 24 RA patients where body composition by upper arm anthropometry closely resembled results achieved using bio-electrical impedance methods. $^{12}$

Body composition by waist measurement has recently been validated against the more cumbersome and time consuming method of underwater weighing and seems to be as accurate as the more traditional method using the sum of four skin folds. ${ }^{9}$ In the female patients we studied there was a correlation with increased acute phase response judged by high ESR or CRP and low FFMI (calculated using waist measurements). These findings are in keeping with a previous study that found that RA patients classified as malnourished had higher ESR and CRP than normally nourished RA patients. ${ }^{13}$

A probable cause for the reduced body mass is weight loss since the onset of disease. A significant proportion of the female group had experienced considerable weight loss since presentation, one in eight had lost more than $15 \%$ of their initial weight. These patients seemed to have a poorer functional outcome as judged by their HAQ scores despite similar disease duration and age to the remainder of the cohort under investigation. In addition patients with the most dramatic weight loss tended to have a higher acute phase response to their disease (although this did not quite reach significance for CRP).

We could find no relation between disease activity and reduced lean body mass in the male population. One possible explanation may be a sampling error because of the smaller size of the male group. Alternatively true differences between the sexes may exist. Hormonal differences could play a part. The reduction of the androgens testosterone and dehydroepiandrosterone seen in patients with RA may be pertinent. ${ }^{14}$ The reduced values seen in men however may still be high enough to afford some protection against lean tissue loss. It may also be relevant that women tend to have a poorer outcome with RA than men $^{15}$ and the greater weight loss seen in the female group may be another marker of their increased morbidity. Further more specific studies in this area are warranted.

The relation between active disease, reduced lean body mass, and weight loss in female patients is probably multifactorial. Although mechanical problems leading to secondary muscle wasting may contribute, this seems unlikely to be an important factor as there is no relation between the HAQ score (a measure of disability and not disease activity) and lower FFMI. The acute phase response seems to be more important, although we did not measure cytokine production they may play a pivotal part in RA cachexia. Interleukin $1 B$ and interleukin 6 both may be systemically increased in RA. ${ }^{17}$ Interleukin 13 may cause reduced appetite while interleukin 6 participates in the production of acute phase reactants by the liver. The role of tumour necrosis factor in weight loss has been studied extensively. Animal studies have shown that infused tumour necrosis factor may cause increased protein degradation. ${ }^{18}$ Higher serum tumour necrosis factor concentrations have been detected in RA patients experiencing a flare of their disease and suffering from cachexia compared with patients without a cachectic flare. ${ }^{12}$ In addition studies in RA and cystic fibrosis patients have shown a relation between raised tumour necrosis factor production and increased resting energy expenditure. ${ }^{1920}$ Thus tumour necrosis factor may lead to increased energy expenditure and increased protein degradation both of which may lead to a reduction in lean body mass.

In conclusion, there is clear evidence from this study that weight loss is a significant problem in female patients with RA and is greatest in those patients with the highest acute phase response to their disease. Women with significant weight loss seem to have a poorer functional outcome.

We are grateful to Miss A Tierney for typing the manuscript and Professor M Lean for his helpful comments.

1 Paget J. Nervous mimicry of organic disease. Lancet 1873;ii:727-9.

2 Higgins M, D'Agostino R, Kannel W, Cobb J. Benefits and adverse effects of weight loss: observations from the Framingham study. Ann Intern Med 1993;119:758-63. 
3 Tellardo JM, Barcia-Sabrido JL, Hanley JA, et al. Predicting mortality based on body composition analysis. Ann Surg 1989;209:81-7.

4 Kotler DP, Tierney AR, Wang J, Pierson RN. Magnitude of body-cell-mass depletion and the timing of death from wasting in AIDS. Am J Clin Nutr 1989;50:444-7.

5 Wilson DO, Rogers RM, Wright EC, Anthonisen NR. Body weight in chronic obstructive airways disease: the National Institute of Health Intermittent Positive Pressure Trial. Am Rev Respir Dis 1992;146:1511-7.

6 Carstairs V, Morris R. Deprivation and health in Scotland. Aberdeen: Aberdeen University Press, 1991.

7 Roubenoff R, Wilson PWF. Advantage of knee height as an index of stature in expression of body composition in adults. Am J Clin Nutr 1993;57:609-13.

8 Frisancho AR. Triceps skinfold and upper arm muscle size norms for assessment of nutritional status. Am J Clin Nutr norms for assessm

9 Lean MEJ, Han TS, Deurenberg P. Predicting body composition by densitometry from simple anthropometric meassition by densitometry from simple anthrop
urements. Am J Clin Nutr 1996;63:4-14.

urements. Am J Clin Nutr 1996;63:4-14.
10 Gregory J, Foster K, Tyler H, Wiseman M. The dietary and nutritional survey of British adults. London: HMSO, 1990.

11 Frisancho AR. New norms of upper limb fat and muscle areas for assessment of nutritional status. Am J Clin Med $1981 ; 34: 2540-5$

12 Rheumatoid cachexia: depletion of lean body mass in rheumatoid arthritis. Possible association with tumour necrosis factor. J Rheumatol 1992;19:1505-10.
13 Helliwell M, Coombes EJ, Moody BJ, Batstone GF, Robertson JC. Nutritional status in patients with rheumatoid arthritis. Ann Rheum Dis 1984;43:386-90.

14 Wilder RL. Neuroendocrine-immune system interactions and autoimmunity. Ann Rev Immunol 1995;13:307-38

15 Leigh JP, Fries JF. Predictors of disability in a longitudinal sample of patients with rheumatoid arthritis. Ann Rheum Dis 1992;51:581-7.

16 Eastgate JA, Wood NC, DiGiovine FS, Symons JA, Grinlinton FM, Duff GW. Correlation of plasma interleukin 1 levels with disease activity in rheumatoid arthritis. Lancet 1988;ii:706-9.

17 Madhok R, Crilly A, Watson J, Capell HA. Serum interleukin 6 levels in rheumatoid arthritis: correlations with clinical and laboratory indices of disease activity. Ann Rheum Dis 1993;52:232-4.

18 Costelli P, Carbo N, Tessitore L, et al. Tumour necrosis factor-alpha mediates changes in tissue protein turnover in a rat cachexia model. J Clin Invest 1993;92:2783-9.

19 Roubenoff R, Roubenoff RA, Cannon JG et al. Rheumatoid cachexia: cytokine-driven hypermetabolism accompanying reduced body cell mass in chronic inflammation. J Clin Invest 1994;93:2379-86.

20 Elborn JS, Cordon SM, Western PJ, MacDonald IA, Shale DJ. Tumour necrosis factor-alpha, resting energy expenditure, cachexia in cystic fibrosis. Clin Sci 1993;85:563-8. 